\title{
Prevalence of Depression and Anxiety in Patients with Dry Eye Syndrome
}

\author{
Nooshin Bazzazi', Mehdi Alizadeh², Mohammad Ahmadpanah ${ }^{3, *}$ (iD), Ali Rooshenas ${ }^{4}$ \\ ${ }^{I}$ Associate Professor, Department of Ophthalmology, School of Medicine, Hamadan University of Medical Sciences, \\ Hamadan, Iran \\ ${ }^{2}$ Assistant Professor, Department of Ophthalmology, School of Medicine, Hamadan University of Medical Sciences, \\ Hamadan, Iran \\ 3 Professor of Cognitive and Clinical Psychology, Research Center for Behavioral Disorders and Substance Abuse, Hamadan \\ University of Medical Sciences, Hamadan, Iran \\ ${ }^{4}$ General Practitioner, Hamadan University of Medical Sciences, Hamadan, Iran
}

* Corresponding Author: Mohammad Ahmadpanah, Research Center for Behavioral Disorders and Substance Abuse, Hamadan University of Medical Sciences, Hamadan, Iran.Email: mlahmad2000@gmail.com

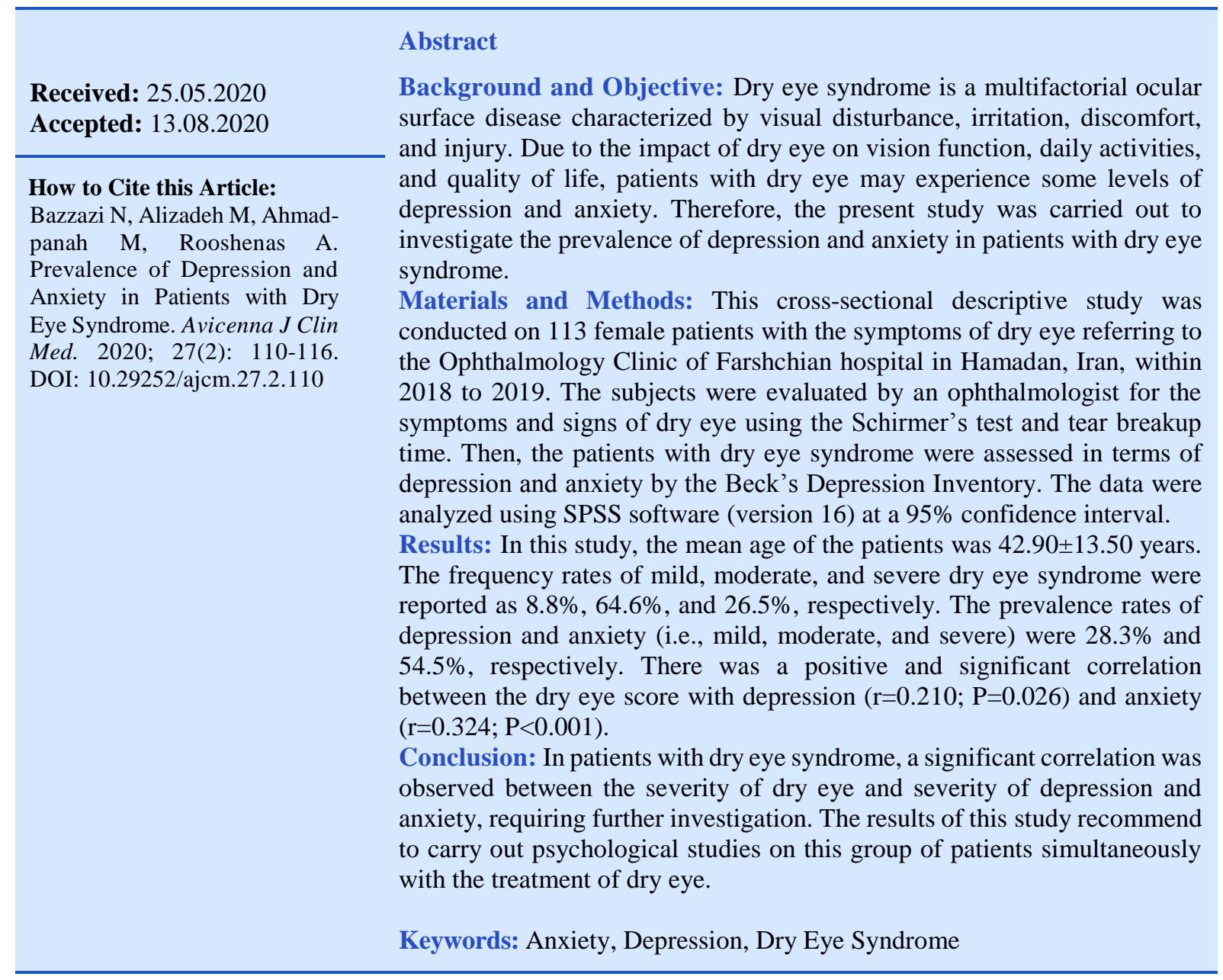




\section{بررسى شيوع افسردى و اضطراب در بيماران مبتلابه سندرم خشكى جشم}

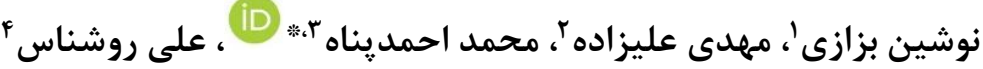

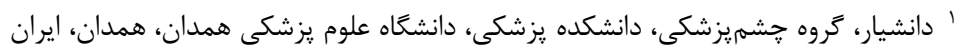

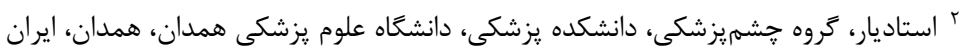

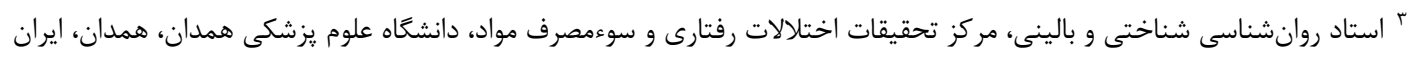

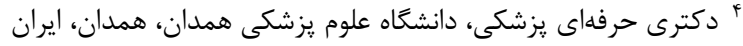

* نويسنده مسئول: محمد احمديناه، مركز تحقيقات اختلالات رفتارى و سوءمصرف مواد، دانشًاه علوم يزشكى همدان، همدان، ايران.

ايميل: m1ahmad2000@gmail.com

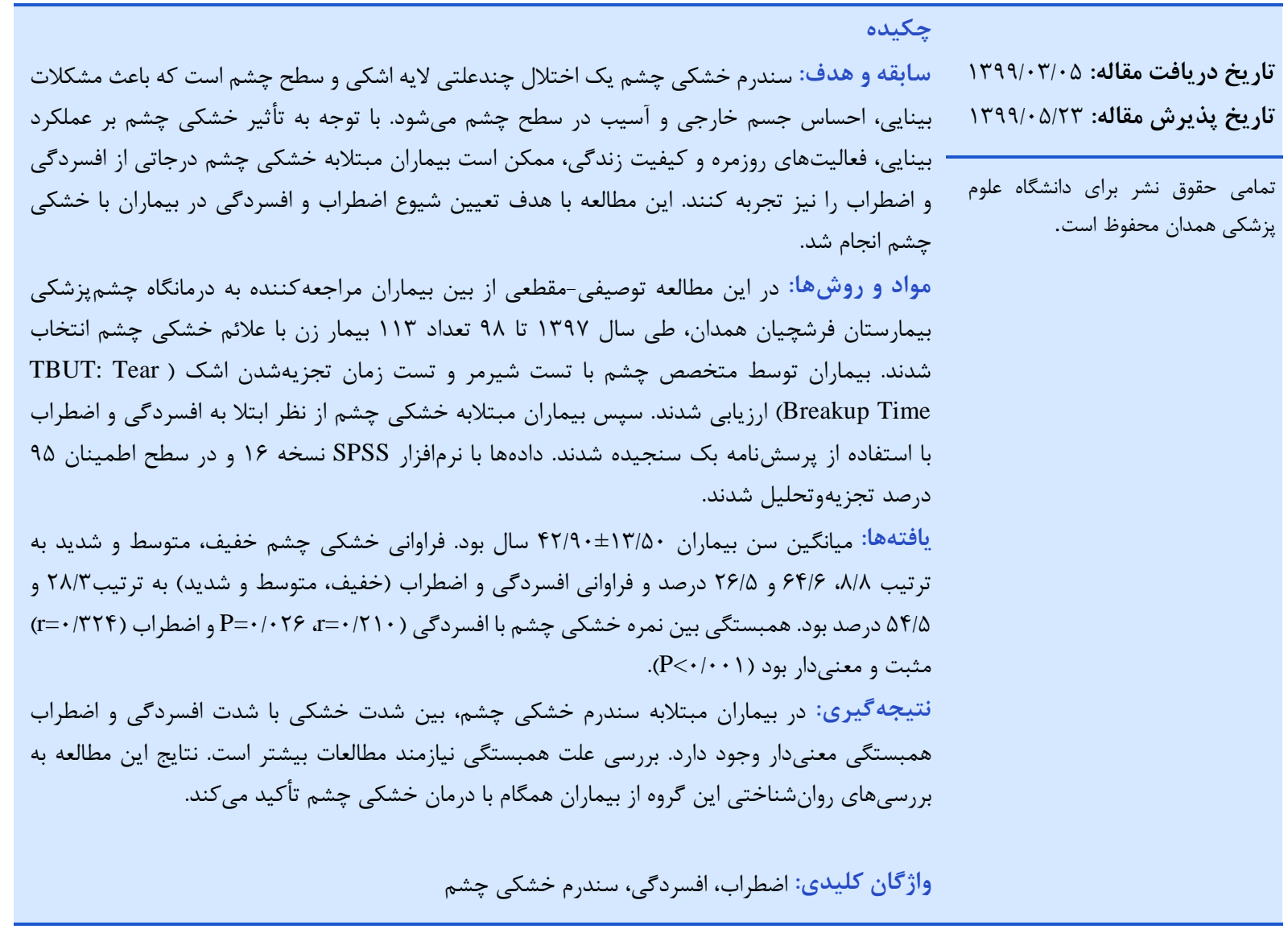

سلامت تبديل شده است. تخمين زده مىشود كه براى كل جمعيت

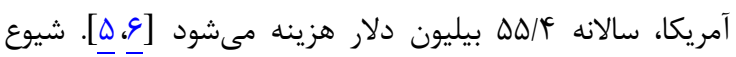

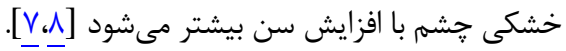

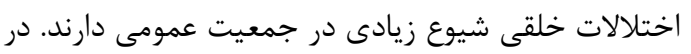

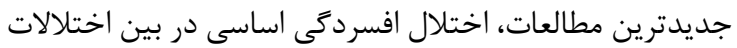

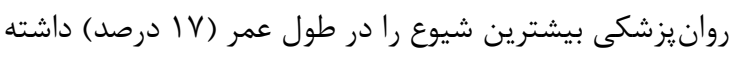

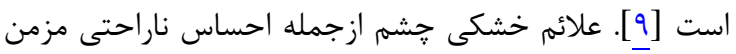

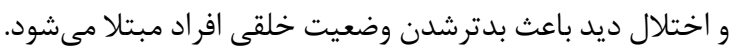

خشكى جشم نوعى بيمارى جندعاملى اشك و سطح جشم

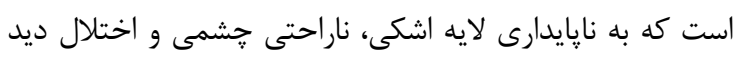

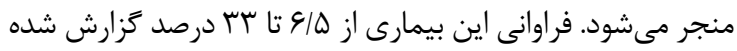

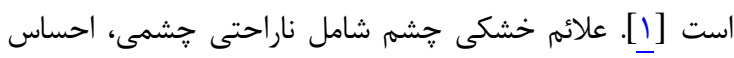

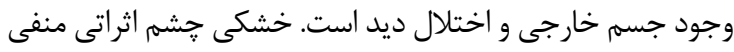

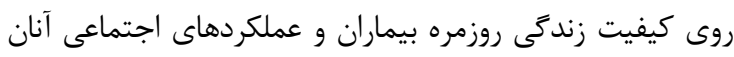

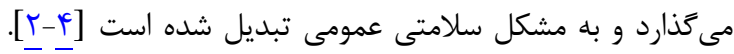

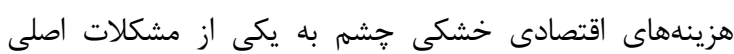


كَزارش شد. يس از تكميلشدن معاينات و تأييد تشخيص بيمارى

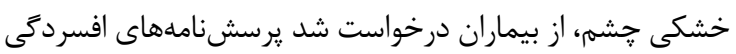
و اضطراب بك را تكميل كنند.

\section{BDI-II: Beck Depression ) برسشنامه افسردكى بك}

Inventory-II

اين برسشنامه شامل إ گ گروه سؤال است. براى نخستين

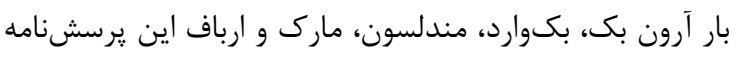

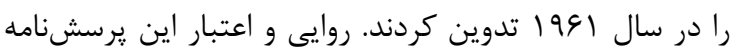

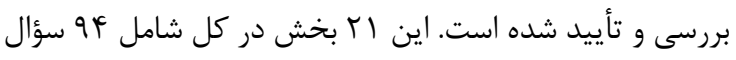

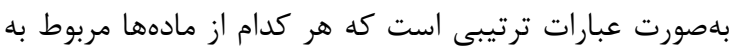

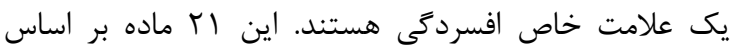
علائمى مثل غمخينى، بدبينى، احساس شكست، نارضايتى،

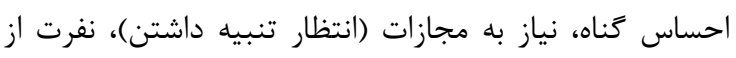

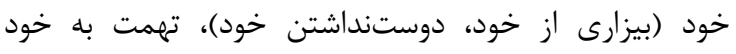

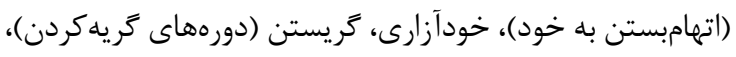

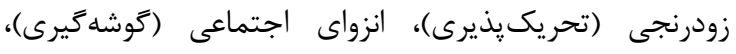

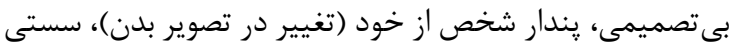

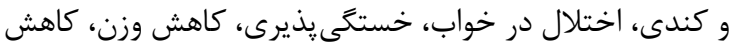

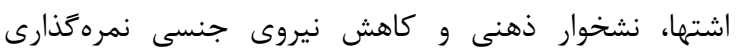

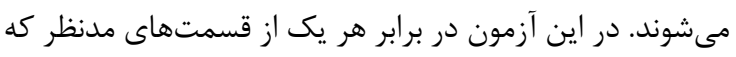

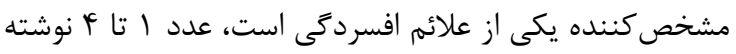

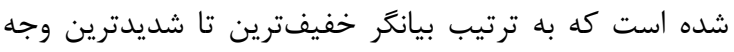

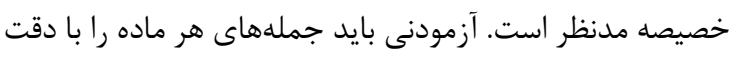

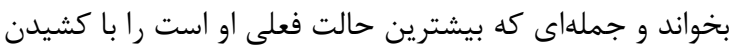
دايره دور آن مشخص كند.

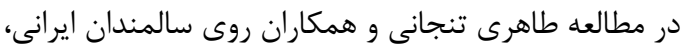

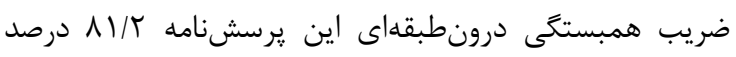

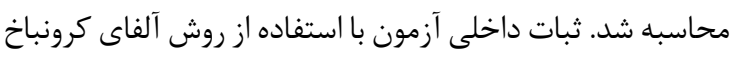

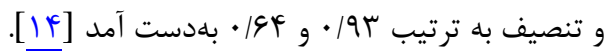

\section{BAI: Beck Anxiety) برسشنامه اضطراب بك}

Inventory

يرسشنامه اضطراب بك يك پرسشنامه خودَزارشى است

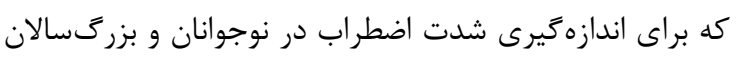

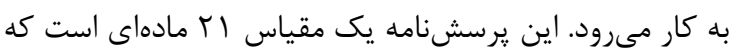

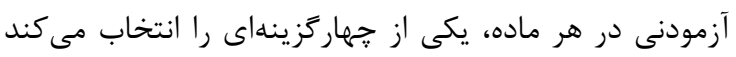

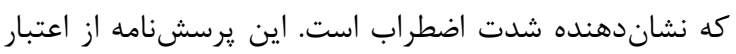

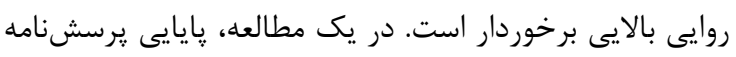

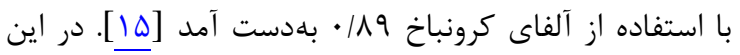

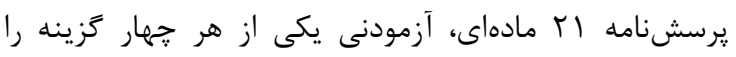

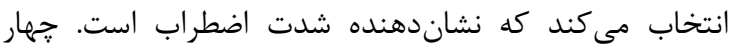

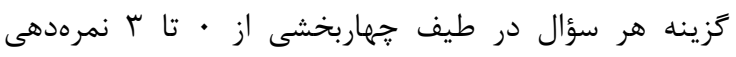

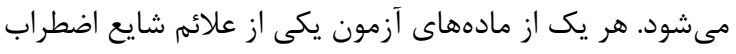

تارى ديدى كه با نايايدارى لايه اشكى ايجاد مىشود، باعث

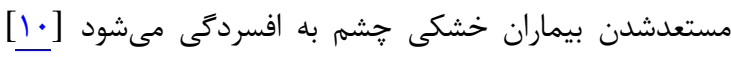

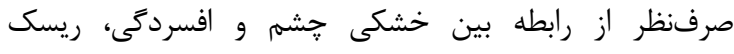

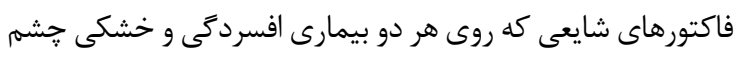

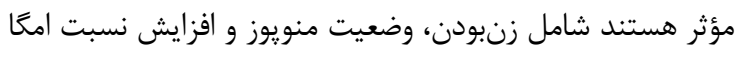

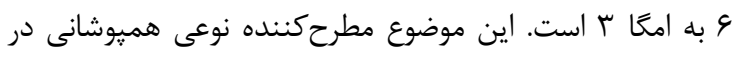

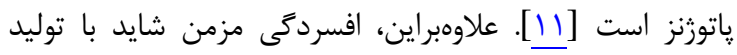

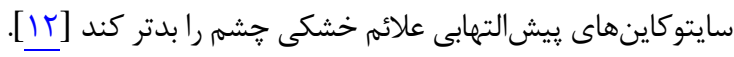

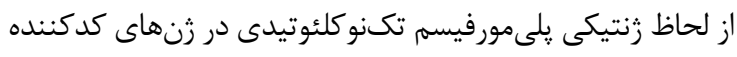

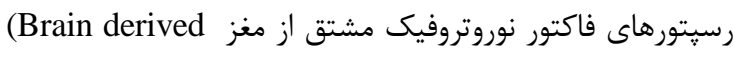
neurotrophic factor) جشم مرتبط هستند [111].

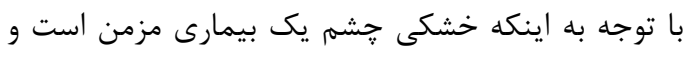

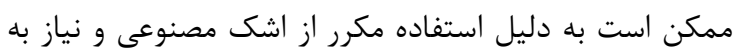

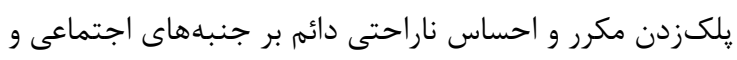

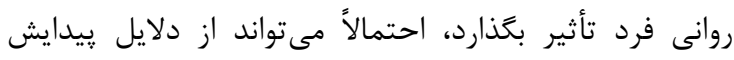

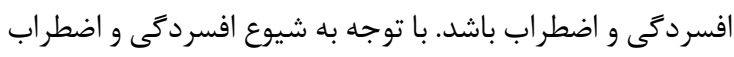

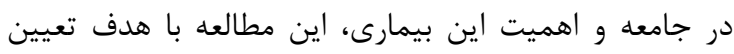

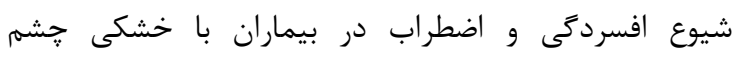

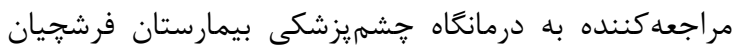
سيناى همدان انجام كرفت.

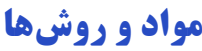

اين مطالعه بهصورت توصيفى-مقطعى طى دوره زمانى

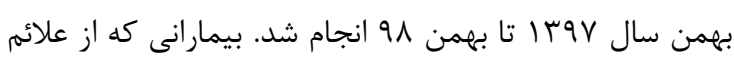
خشكى جشم شاكى بودند، به روش نمونه ميرى در دسترس و

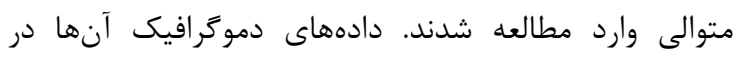

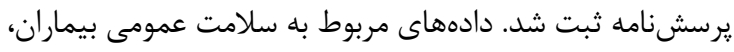

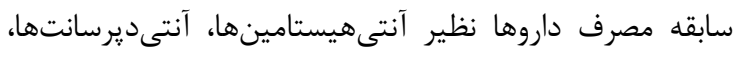

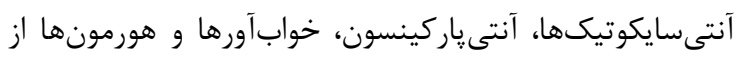

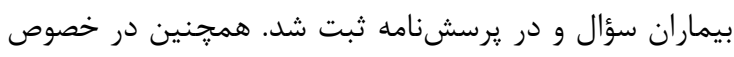

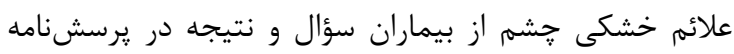

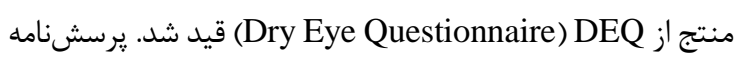

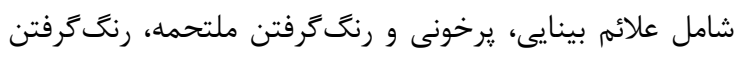

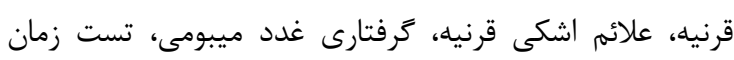

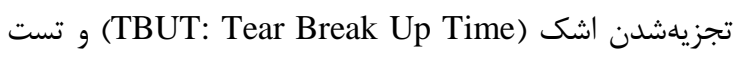

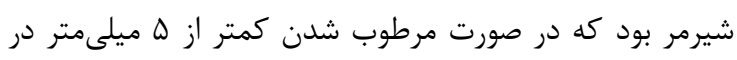

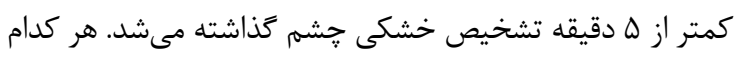

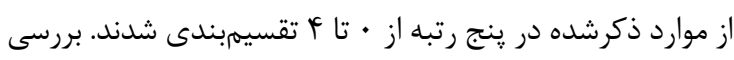

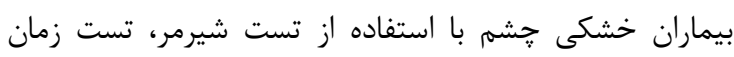

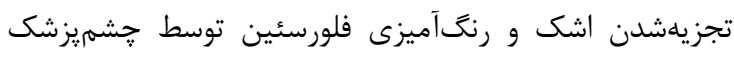

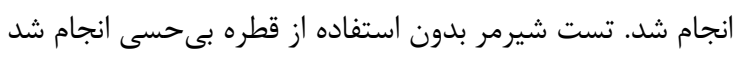

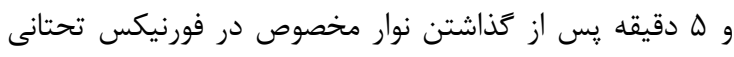

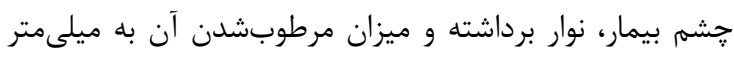


جدول ا: توزيع فراوانى افسردگى و اضطراب در بيماران مطالعهشده با خشكى خشم و اصطراب

\begin{tabular}{|c|c|c|}
\hline درصد & تعداد & متغير \\
\hline & & افسردگى \\
\hline$V \backslash / V$ & 11 & ن نرمال \\
\hline$I V / V$ & $r \cdot$ & خفيف \\
\hline$\Lambda / \Lambda$ & 1 . & متوسط \\
\hline $1 / 1$ & r & شديد \\
\hline $1 \cdots$ & $11 \pi$ & مجموع \\
\hline & & اضطراب \\
\hline$Y Q / 1$ & $\Delta 1$ & نرمال \\
\hline$F Y / D$ & is & خفيف \\
\hline$N / \cdot$ & 9 & متوسط \\
\hline 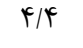 & $\Delta$ & شديد \\
\hline $1 \cdots$ & 114 & مجموع \\
\hline
\end{tabular}

بر اساس نتيجه آزمون ضريب همبستكى يرسون،

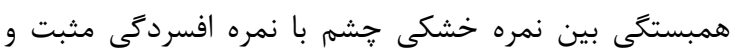

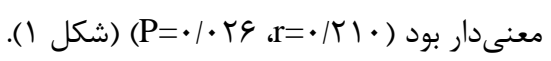

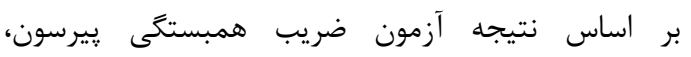

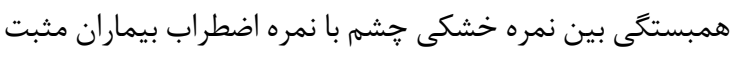

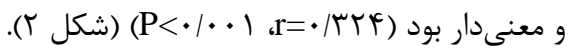

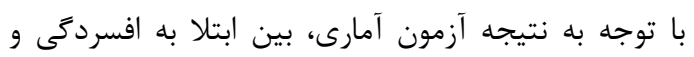

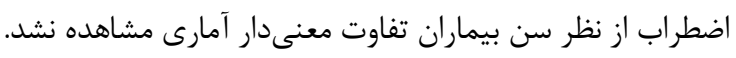

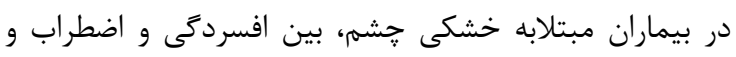

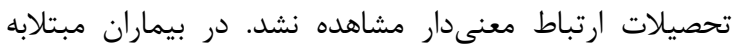

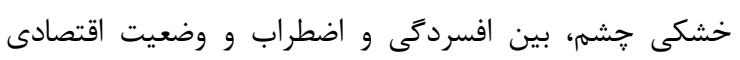
ارتباط معنى دار مشاهده نشد.
(علائم ذهنى، بدنى و هراس) را توصيف مى كند. نمره كل اين

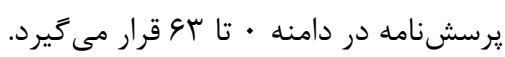

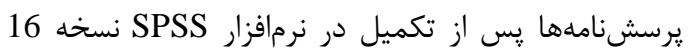

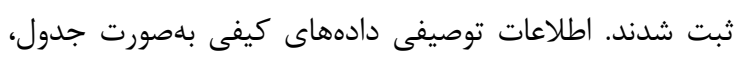

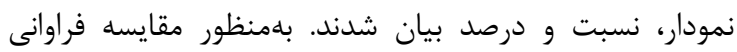

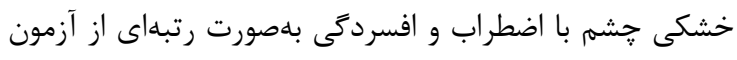

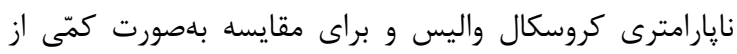

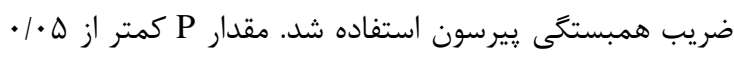

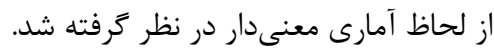

\section{كافْته كا}

در اين مطالعه سا || بيمار بررسى شدند. از نظر جنسيت

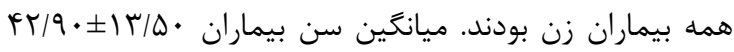

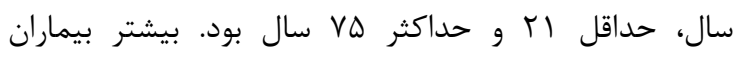
تحصيلات دييلم (N/A V./ درصد) و وضعيت اقتصادى ضعيف

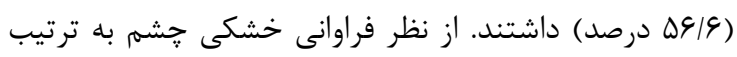

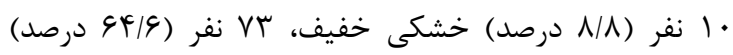

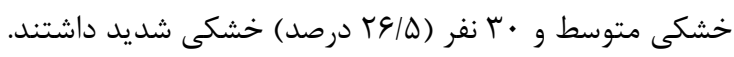

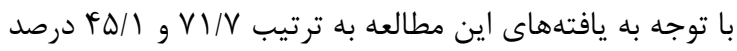

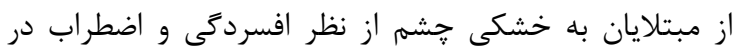

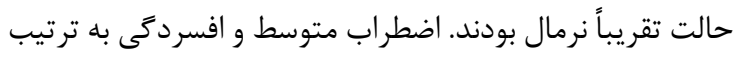

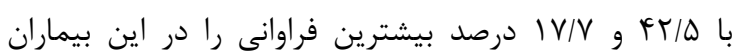
داشتند (جدول ()).

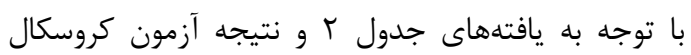

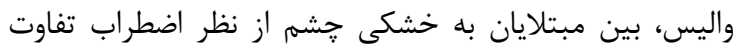

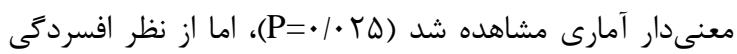

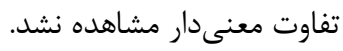

جدول r: توزيع فراوانى خشكى جشم بر حسب افسردگى و اضطراب در بيماران مطالعهشده

\begin{tabular}{|c|c|c|c|c|}
\hline $\mathbf{P}$ & شديد & مشتوسط جشى & خفيف & متغير \\
\hline . ITMF & $\begin{array}{l}(\varepsilon \cdot / \cdot) \backslash \wedge \\
(r / r) \bigvee \\
(\mid r / r) Y \\
(r / r) \backslash \\
(1 \cdot \cdot) r \cdot\end{array}$ & $\begin{array}{c}(V \Delta / r) \Delta \Delta \\
(\mid \varepsilon / \mathcal{F}) \mid r \\
(\varepsilon / \Lambda) \Delta \\
(1 / \mathcal{F}) \mid \\
(1 \cdots) V r\end{array}$ & $\begin{array}{c}(\Lambda \cdot / \cdot) \Lambda \\
(1 \cdot / \cdot)) \\
(1 \cdot / \cdot)) \\
(\cdot) \cdot \\
(1 \cdot \cdot)) !\end{array}$ & خرمال \\
\hline$\cdot / \cdot r \Delta$ & 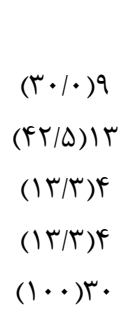 & 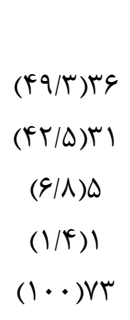 & $\begin{array}{c}(\xi \cdot / \cdot)^{q} \\
(\xi \cdot / \cdot)^{f} \\
(\cdot) \cdot \\
(\cdot) \cdot \\
(1 \cdot \cdot)) \cdot\end{array}$ & خرمال \\
\hline
\end{tabular}




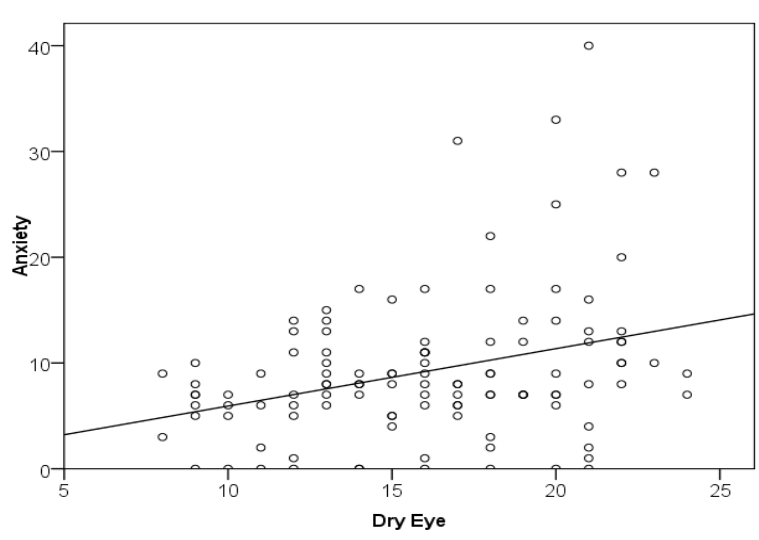

شكل r: ضريب همبستكى بين نمره خشكى حشم با اضطراب در

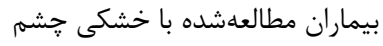

معنى دار مشاهده شد. در خصوص ارتباط بين سن و خشكى نشى

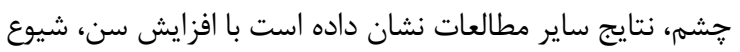

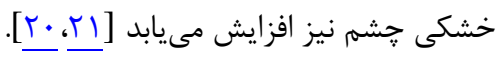
در مطالعات يزوهشى مرورى اصيل و متاآناليز صورت ترفتيه،

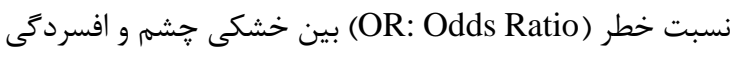

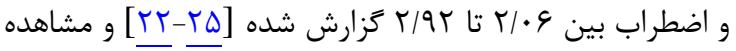

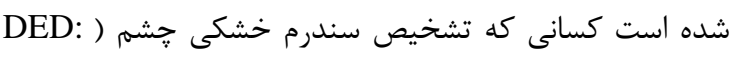
(Dry Eye Disease استرسهاى سايكولوزيك، خلق افسرده، مشكلات اضطرابى و

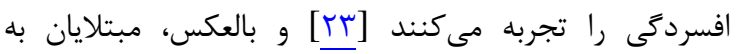

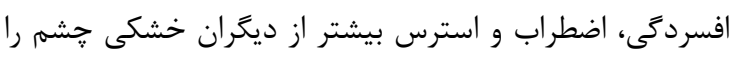

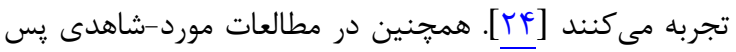

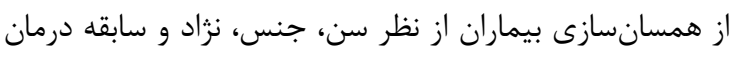

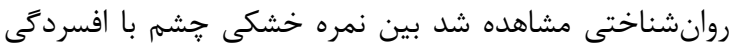

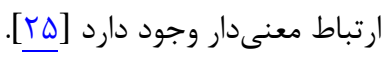

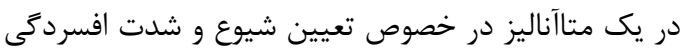

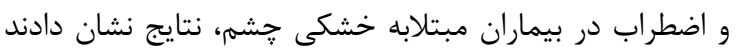

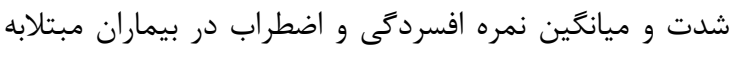

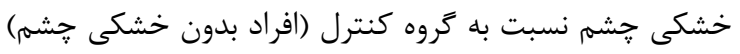

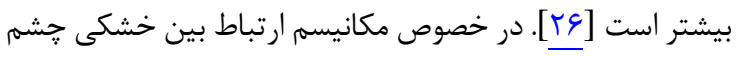

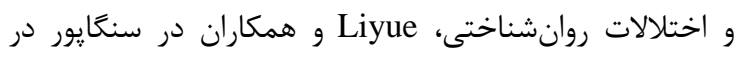

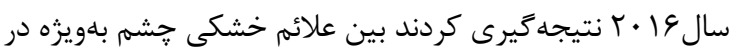
بيمارانى كه دفعات بيشترى تارى ديد ايجاد مى كند، با علائم

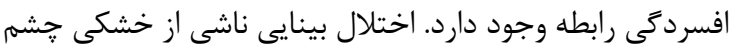

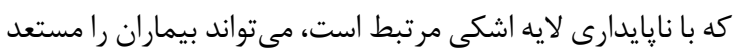

افسردگى كند [19].

\section{نتيجه تيرى}

اين مطالعه نشان داد در بيماران مبتلابه خشكى حشمه، بين

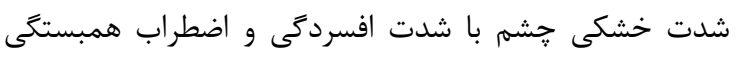
معنى دارى وجود دارد. در خصوص ارتباط بين خشكى جشى جشم و

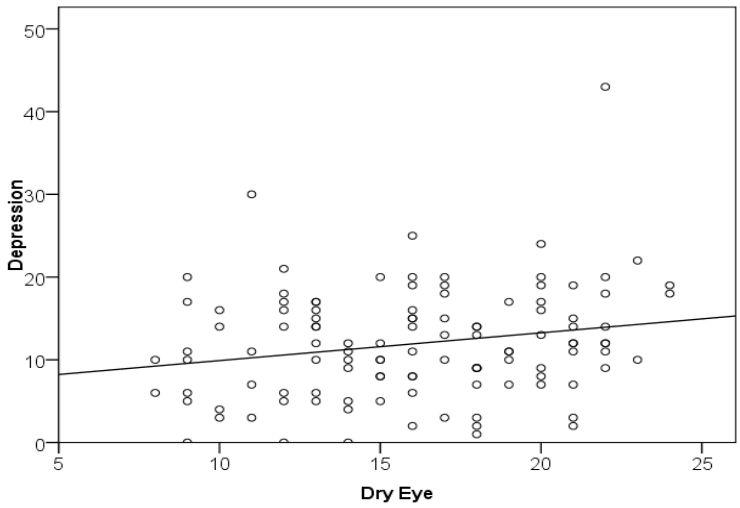

$$
\text { شكل ا: ضريب همبستگى بين نمره خشكى خشم با افسردگى در }
$$

بج

نتايج مطالعه حاضر نشان داد در بيماران مبتلابه خشكى

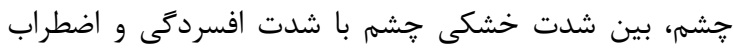

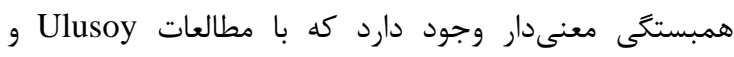

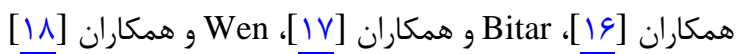
و و و همكاران [19yue] همسو است.

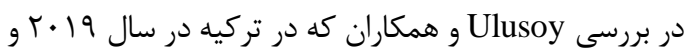

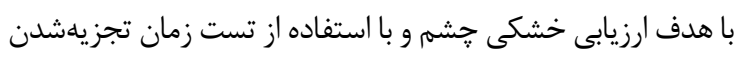

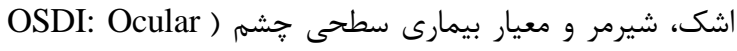
(Surface Disease Index

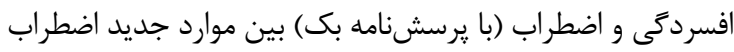

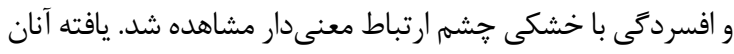

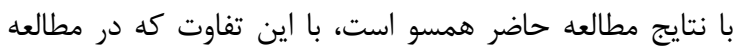

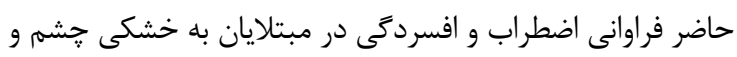

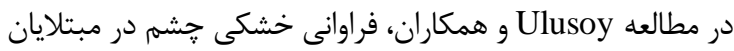

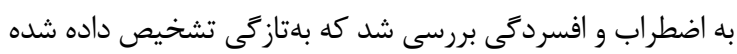

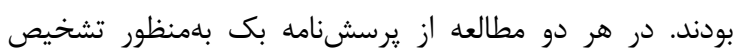
افسردگى و اضطراب استفاده شده است.

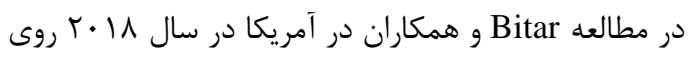

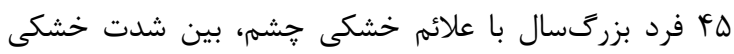

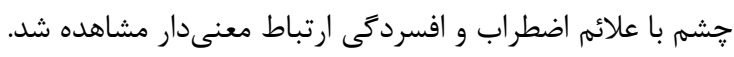
حجم نمونه مطالعه حاضر بيشتر از مطالعه يادشده بود، اما نتايج دو مطالعه همسو است.

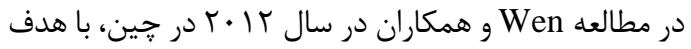
تعيين فراوانى خشكى جشم و ريسك فاكتورهاى

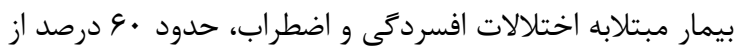

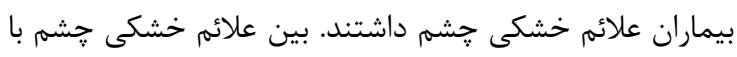

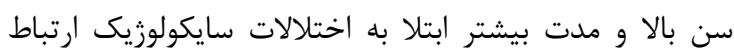
معنى دار مشاهده شد. در مطالعه حاضر بهجاى ارئ ارزيابى علائم خشكى جشم در مبتلايان به اختلالات سايكولوزيك، علائم

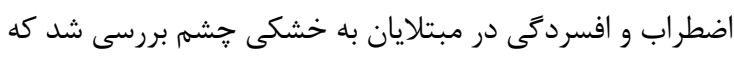

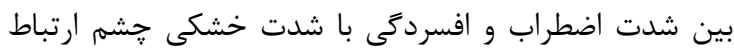


1) شناسه IR.UMSHA.REC.1398.055 مطالعه حاضر تأييد كرده است. ييش از شروع مطالعه، ضمن توضيح اهداف

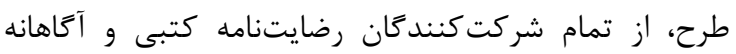
كرفته شد.

سمهم ذويسندكان

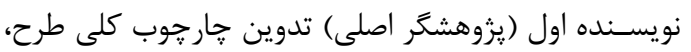

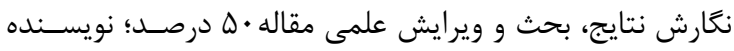

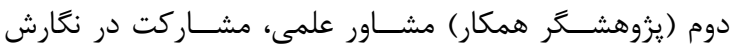

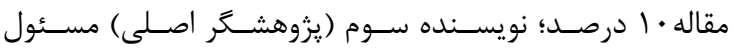

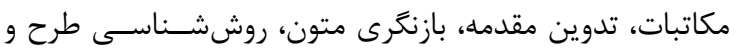

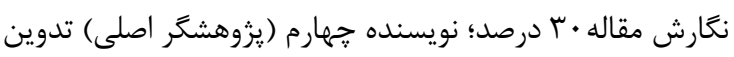

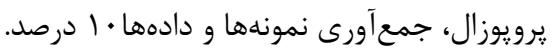

\section{حمايت مالى}

اين يروزه از سوى معاونت تحقيقات و فناورى دانشعاه علوم يزشكى همدان يشتيبانى مالى شده است.

\section{REFERENCES}

1. Janine AS. The epidemiology of dry eye disease: report of the epidemiological subcommittee of the international dry eye workshop. Ocul Surf. 2007;5(2):93-107. PMID: 17508117 DOI: 10.1016/s1542-0124(12)70082-4

2. Mertzanis P, Abetz L, Rajagopalan K, Espindle D, Chalmers $\mathrm{R}$, Snyder C, et al. The relative burden of dry eye in patients' lives: comparisons to a US normative sample. Invest Ophthalmol Vis Sci. 2005;46(1):46-50. PMID: 15623753 DOI: 10.1167/iovs.03-0915

3. Miljanović B, Dana R, Sullivan DA, Schaumberg DA. Impact of dry eye syndrome on vision-related quality of life. Am J Ophthalmol. 2007;143(3):409-15. PMID: 17317388 DOI: 10.1016/j.ajo.2006.11.060

4. Daly EJ, Trivedi MH, Wisniewski SR, Nierenberg AA, Gaynes BN, Warden D, et al. Health-related quality of life in depression: a STAR*D report. Ann Clin Psychiatry. 2010;22(1):43-55. PMID: 20196982

5. Kalangara JP, Galor A, Levitt RC, Felix ER, Alegret R, Sarantopoulos CD. Burning eye syndrome: do neuropathic pain mechanisms underlie chronic dry eye? Pain Med. 2016;17(4):746-55. PMID: 26814296 DOI: $10.1093 / \mathrm{pm} /$ pnv070

6. Pepose JS, Sullivan BD, Foulks GN, Lemp MA. The value of tear osmolarity as a metric in evaluating the response to dry eye therapy in the clinic and in clinical trials. Am J Ophthalmol. 2014;157(1):4-6.e1. PMID: 24220266 DOI: 10.1016/j.ajo.2013.10.020

7. Schein OD, Muñoz B, Tielsch JM, Bandeen-Roche K, West S. Prevalence of dry eye among the elderly. Am $J$ Ophthalmol. 1997;124(6):723-8. PMID: 9402817 DOI: 10.1016/s0002-9394(14)71688-5

8. Galor A, Feuer W, Lee DJ, Florez H, Carter D, Pouyeh B, et al. Prevalence and risk factors of dry eye syndrome in a United States veterans affairs population. Am J Ophthalmol. 2011;152(3):377-84.e2. PMID: 21684522 DOI: 10.1016/j. ajo.2011.02.026

9. Galor A, Feuer W, Lee DJ, Florez H, Faler AL, Zann KL, et al. Depression, post-traumatic stress disorder, and dry eye syndrome: a study utilizing the national United States Veterans Affairs administrative database. Am J Ophthalmol. 2012;154(2):340-6.e2. PMID: 22541654 DOI: 10.1016/j. ajo.2012.02.009

10. Kim KW, Han SB, Han ER, Woo SJ, Lee JJ, Yoon JC, et al.

$$
\begin{aligned}
& \text { اختلالات روانشناختى به دليل ماهيت مطالعه حاضر، وجود } \\
& \text { همزمانى خشكى جشم با اختلالات اضطرابى و افسردگى لزوماً } \\
& \text { نشاندهنده ارتباط عليتى نيست و اين موضوع به مطالعه و بررسى } \\
& \text { بيشترى نياز دارد. }
\end{aligned}
$$

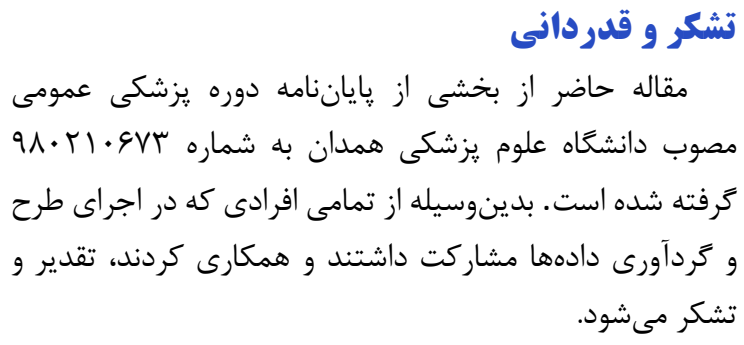

تضاد منافع

هيجزَنه تعارض منافعى بين نويسندگان و نتايج مطالعه

$$
\text { وجود ندارد. }
$$

\section{ملاحظات اخلاقى}

كميته اخلاق در يزوهش دانشكاه علوم يزشكى همدان با بال

Association between depression and dry eye disease in an elderly population. Invest Ophthalmol Vis Sci. 2011; 52(11):7954-8. PMID: 21896858 DOI: 10.1167/iovs.11$\underline{8050}$

11. Miljanovic B, Trivedi KA, Dana MR, Gilbard JP, Buring JE, Schaumberg DA. Relation between dietary n-3 and n-6 fatty acids and clinically diagnosed dry eye syndrome in women. Am J Clin Nutr. 2005;82(4):887-93. PMID: 16210721 DOI: 10.1093/ajcn/82.4.887

12. Lutgendorf SK, Garand L, Buckwalter KC, Reimer TT, Hong SY, Lubaroff DM. Life stress, mood disturbance, and elevated interleukin-6 in healthy older women. $J$ Gerontol A Biol Sci Med Sci. 1999;54(9):M434-9. PMID: 10536645 DOI: $10.1093 /$ gerona/54.9.m434

13. Hallak JA, Tibrewal S, Mohindra N, Gao X, Jain S. Single nucleotide polymorphisms in the BDNF, VDR, and DNASE 1 genes in dry eye disease patients: a case-control study. Invest Ophthalmol Vis Sci. 2015;56(10):5990-6. PMID: 26393465 DOI: $10.1167 /$ iovs. $15-17036$

14. Hamidi R. Validity and reliability Beck depression inventoryII among the Iranian elderly population. J Sabzevar Univ Med Sci. 2015;22(1):189-98. [Persian]

15. Salari-Moghaddam S, Ranjbar AR, Fathi-Ashtiani A. Validity and reliability measurement of the Persian version of anxiety control questionnaire. J Clin Psycol. 2018;9(4):3343. DOI: $10.22075 / \mathrm{JCP} .2018 .11010 .1073$

16. Ulusoy MO, Ișık-Ulusoy S, Kıvanç SA. Evaluation of dry eye disease in newly diagnosed anxiety and depression patients using anterior segment optical coherence tomography. Eye Vis. 2019;6(1):25. PMID: 31417939 DOI: 10.1186/s40662019-0149-y

17. Bitar MS, Olson DJ, Li M, Davis RM. The correlation between dry eyes, anxiety and depression: the Sicca, anxiety and depression study. Cornea. 2019;38(6):684-9. PMID: 30950896 DOI: 10.1097/ICO.0000000000001932

18. Wen W, Wu Y, Chen Y, Gong L, Li M, Chen X, et al. Dry eye disease in patients with depressive and anxiety disorders in Shanghai. Cornea. 2012;31(6):686-92. PMID: 22382595 DOI: $10.1097 / \mathrm{ICO} .0 \mathrm{~b} 013 \mathrm{e} 3182261590$

19. Liyue H, Chiang PP, Sung SC, Tong L. Dry eye-related visual blurring and irrelative symptoms and their association with depression and anxiety in eye clinic patients. Curr Eye Res. 2016;41(5):590-9. PMID: 26337790 DOI: 10.3109/02713 
683.2015.1056804

20. Schaumberg DA, Dana R, Buring JE, Sullivan DA. Prevalence of dry eye disease among US men: estimates from the physicians' health studies. Arch Ophthalmol. 2009; 127(6):763-8. PMID: 19506195 DOI: 10.1001/archoph thalmol.2009.103

21. Schaumberg DA, Sullivan DA, Buring JE, Dana MR. Prevalence of dry eye syndrome among US women. Am J Ophthalmol. 2003;136(2):318-26. PMID: 12888056 DOI: 10.1016/s0002-9394(03)00218-6

22. Wan K, Chen L, Young A. Depression and anxiety in dry eye disease: a systematic review and meta-analysis. Eye. 2016;30(12):1558-67. PMID: 27518547 DOI: 10.1038/eye. 2016.186

23. Na KS, Han K, Park YG, Na C, Joo CK. Depression, stress, quality of life, and dry eye disease in Korean women: a population-based study. Cornea. 2015;34(7):733-8. PMID: 26002151 DOI: $10.1097 / \mathrm{ICO} .0000000000000464$

24. Yilmaz U, Gokler ME, Unsal A. Dry eye disease and depression-anxiety-stress: a hospital-based case control study in Turkey. Pak J Med Sci. 2015;31(3):626-31. PMID: 26150857 DOI: $10.12669 /$ pims.313.7091

25. Hallak JA, Tibrewal S, Jain S. Depressive symptoms in dry eye disease patients: a case-control study using the Beck Depression Inventory. Cornea. 2015;34(12):1545-50. PMID: 26426334 DOI: $10.1097 / I C O .0000000000000641$

26. Labbé A, Wang YX, Jie Y, Baudouin C, Jonas JB, Xu L. Dry eye disease, dry eye symptoms and depression: the Beijing Eye Study. Br J Ophthalmol. 2013;97(11):1399-403. PMID: 24013959 DOI: 10.1136/bjophthalmol-2013-303838 